Pacific Journal of Mathematics

STOCHASTIC CONVEX PROGRAMMING: BASIC DUALITY

AND ROGER JEAN-BAPTISTE ROBERT WE 


\title{
STOCHASTIC CONVEX PROGRAMMING: BASIC DUALITY
}

\author{
R. T. Rockafellar And R. J.-B. Wets
}

A duality theory is developed for stochastic programs with convex objective and convex constraints. The problem consists in selecting $x_{1} \in R^{n_{1}}$ and $x_{2} \in \mathscr{L}^{\infty}\left(S, \Sigma, \sigma ; R^{n_{2}}\right)$ so as to satisfy the constraints and minimize total expected cost, where $\sigma$ is a probability measure and the constraints as well as the objective are functions of the random elements of the problem. Under the additional restriction that $x_{1}$ and $x_{2}(s)$ belong to compact subsets of $R^{n_{2}}$ and $R^{n_{2}}$ respectively, it is shown that the problem is equivalent to the more common dynamic formulation for stochastic programs with recourse, a basic duality theorem - of the type $\min =\sup -$ is proved and qualitative results on the existence of dual solutions are derived.

1. Introduction. In this paper we study a two-stage stochastic optimization problem associated with the following heuristic model. First, a vector $x_{1}$ in $R^{n_{1}}$ is chosen subject to the constraints

$$
x_{1} \in C_{1} \quad \text { and } \quad f_{11}\left(x_{1}\right) \leqq 0, \quad i=1, \cdots, m_{1} \text {, }
$$

at a cost represented by the expression $f_{10}\left(x_{1}\right)$. Next an element $s$ of $S$ is "observed", where $(S, \Sigma, \sigma)$ is a probability space. Finally, a vector $x_{2}(s)$ in $R^{n_{2}}$ is chosen subject to the constraints

$$
x_{2}(s) \in C_{2} \quad \text { and } \quad f_{2 i}\left(s, x_{1}, x_{2}(s)\right) \leqq 0, \quad i=1, \cdots, m_{2},
$$

at a cost $f_{20}\left(s, x_{1}, x_{2}(s)\right)$. The problem is to choose $x_{1}$ and the function $x_{2}(\cdot)$ so as to minimize the total expected cost

$$
f_{10}\left(x_{1}\right)+\int_{S} f_{20}\left(s, x_{1}, x_{2}(s)\right) \sigma(d s) \text {. }
$$

This is a stochastic programming problem with recourse; the function $x_{2}(\cdot)$ specifies the recourse decision.

To make the formulation rigorous, one needs, besides appropriate assumptions on the sets $C_{k}$ and functions $f_{k i}$, some restriction on the class of recourse functions which are admitted, in particular in ensuring that the expected cost (1.3) is in some sense well-defined. For technical reasons, we concentrate below on the case where $x_{2}(s)$ is an essentially 
bounded, measurable function of $s \in S$, and the second-stage constraint (1.2) is only required to hold almost surely (i.e. except for $s$ in a subset of $S$ of $\sigma$-measure zero). However, some justification of this restriction is offered in $\S 3$, and it is shown that, at least when $C_{2}$ is bounded, no real generality is lost thereby.

Our main purpose is to exploit the possibilities of the convex case, where everything is convex in $x_{1}$ and $x_{2}$. We introduce a Lagrangian function in terms of multipliers for the first-stage and second-stage constraints. This leads to a saddle-point condition for optimality which is sufficient and "almost" necessary. The optimal multipliers are solutions to a certain dual problem and can be interpreted as "equilibrium prices" relative to certain perturbations. The dual problem furnishes a description of the directional derivatives of the infimum in the original problem with respect to the perturbation parameters. The vehicle for this analysis is the general "perturbational" theory of duality for convex optimization problems [7], [8], and various results on convex integral functionals and their conjugates [9], [10], [11].

The following technical assumptions are in force throughout the paper. The sets $C_{1}$ and $C_{2}$ are convex, closed and nonempty. The functions $f_{1 i}$ on $R^{n_{1}}$ and $f_{2 t}(s, \cdot, \cdot)$ on $R^{n_{1}} \times R^{n_{2}}$ are convex, everywheredefined and finite (hence continuous). Furthermore, for each $\left(x_{1}, x_{2}\right)$ in $R^{n_{1}} \times R^{n_{2}}$ the functions $f_{2 l}\left(\cdot, x_{1}, x_{2}\right)$ are measurable on $S$, in fact summable for $i=0$ and bounded for $i=1, \cdots, m_{2}$. These assumptions imply that if $x_{1}: S \rightarrow R^{n_{1}}$ and $x_{2}: S \rightarrow R^{n_{2}}$ are arbitrary bounded, measurable functions, then the functions

$$
s \rightarrow f_{2 l}\left(s, x_{1}(s), x_{2}(s)\right), \quad i=0,1, \cdots, m_{2},
$$

are measurable, in fact summable for $i=0$ and essentially bounded for $i=1, \cdots, m_{2}$; see [11, Theorem 2] and [10, Theorem 4]. In particular, the cost expression (1.3) represents well-defined real number if $x_{2}(s)$ is a bounded, measurable function of $s \in S$.

Duality is developed by embedding the problem in a class of "perturbed" problems. Let

$$
X=R^{n_{1}} \times \mathscr{L}_{n_{2}}^{\infty} \quad \text { and } \quad U=R^{m_{1}} \times \mathscr{L}_{m_{2}}^{\infty},
$$

where $\mathscr{L}_{n}^{p}$ denotes the usual Lebesgue space of $R^{n}$-valued functions over $(S, \Sigma, \sigma)$. The function

$$
F: X \times U \rightarrow R \cup\{+\infty\}
$$

is defined as follows. If $x=\left(x_{1}, x_{2}\right) \in X$ and $u=\left(u_{1}, u_{2}\right) \in U$ satisfy

$$
x_{1} \in C_{1} \quad \text { and } \quad f_{1 i}\left(x_{1}\right) \leqq u_{1}, \quad i=1, \cdots, m_{1},
$$


and almost surely

$$
x_{2}(s) \in C_{2} \quad \text { and } \quad f_{2 i}\left(s, x_{1}, x_{2}(s)\right) \leqq u_{2 i}(s), \quad i=1, \cdots, m_{2},
$$

then $F(x, u)$ is the expected cost (1.3); otherwise $F(x, u)=+\infty$. We denote by $\mathbf{P}(u)$ the problem of minimizing $F(x, u)$ over all $x \in X$, i.e. the problem of minimizing (1.3) over all $x_{1} \in R^{n_{1}}$ and $x_{2} \in \mathscr{L}_{n_{2}}^{\infty}$ satisfying (1.4) and almost surely (1.5). The problem $\mathbf{P}(C)$, corresponding to the original model, is denoted simply by $\mathbf{P}$. The problems all make sense technically, in view of the remarks above.

The system of perturbations can lead to different forms of the dual problem and Lagrangian, depending on how a space $Y$ is paired with $U$. Here we take

$$
\langle u, y\rangle=u_{1} \cdot y_{1}+\int_{S} u_{2}(s) \cdot y_{2}(s) \sigma(d s) \text { for } y \in Y=R^{m_{1}} \times \mathscr{L}_{m_{2}}^{1} .
$$

We remark, however, that complementary results of considerable interest can be obtained if $\mathscr{L}_{m_{2}}^{1}$ is replaced in this pairing by the dual Banach space $\left(\mathscr{L}_{m_{2}}^{\infty}\right)^{*}$. These results will be treated in a subsequent paper.

According to general theory [8], the Lagrangian associated with the system of perturbations $u \in U$ is the function:

$$
L: X \times Y \rightarrow R \cup\{ \pm \infty\}
$$

defined by

$$
L(x, y)=\inf _{u \in U}\{\langle u, y\rangle+F(x, u)\}
$$

In the case at hand, with the pairing given by (1.6), it is easy to calculate that

$$
\begin{aligned}
L(x, y)= & L_{1}\left(x_{1}, y_{1}\right)+\int_{s} L_{2}\left(s, x_{1}, x_{2}(s), y_{2}(s)\right) \sigma(d s) \\
& \text { if } x \in X_{0} \text { and } y \in Y_{0}, \\
= & -\infty \text { if } x \in X_{0} \text { and } y \notin Y_{0}, \\
= & +\infty \text { if } x \notin X_{0},
\end{aligned}
$$

where

(1.9) $X_{0}=\left\{x=\left(x_{1}, x_{2}\right) \in X \mid x_{1} \in C_{1}\right.$ and almost surely $\left.x_{2}(s) \in C_{2}\right\}$

(1.10) $Y_{0}=\left\{y=\left(y_{1}, y_{2}\right) \in Y \mid y_{1} \geqq 0\right.$ and almost surely $\left.y_{2}(s) \geqq 0\right\}$, 
(1.11) $L_{1}\left(x_{1}, y_{1}\right)=f_{10}\left(x_{1}\right)+\sum_{i=1}^{m_{1}} y_{1 i} f_{1 i}\left(x_{1}\right)$,

(1.12) $L_{2}\left(s, x_{1}, x_{2}, y_{2}\right)=f_{20}\left(s, x_{1}, x_{2}\right)+\sum_{i=1}^{m_{2}} y_{2 i} f_{2 l}\left(s, x_{1}, x_{2}\right)$.

Note that $L(x, y)$ is convex in $x \in X$ and concave in $y \in Y$.

From formula (1.7), one also calculates at once that

$$
F(x, u)=\sup _{y \in Y}\{L(x, y)-\langle u, y\rangle\} \quad \text { for all } x \in X, u \in U
$$

In particular, the essential objective function in $\mathbf{P}$ is represented by

$$
F(x, 0)=\sup _{y \in Y} L(x, y)
$$

and the optimal value in $\mathbf{P}$ is therefore

$$
\inf \mathbf{P}=\inf _{x \in X} \sup _{y \in Y} L(x, y) .
$$

We define the dual of $\mathbf{P}$ to be the problem: $\mathbf{D}$ maximize $g(y)$ over all $y \in Y$, where $g(y)=\inf _{x \in X} L(x, y)$. The function $g$ is concave, since $L(x, y)$ is concave in $y$. The optimal value in $\mathbf{D}$ is

$$
\sup \mathbf{D}=\sup _{y \in Y} \inf _{x \in X} L(x, y)
$$

and properties relating the optimal values and optimal solutions of $\mathbf{P}$ and D are minimax properties of the Lagrangian $L$. For example, the following fact is a simple consequence of the definition of $\mathbf{D}$ and the representation (1.14) of the essential objective function in $\mathbf{P}$ (cf. [8, Theorem 2]):

The pair $(x, y) \in X \times Y$ is a saddle-point of $L$ if and only if $x$ gives the minimum in $\mathbf{P}, y$ gives the maximum in $\mathbf{D}$, and $\min \mathbf{P}=\max \mathbf{D}$ (finite).

If a duality theorem of the type inf $\mathbf{P}=\max \mathbf{D}$ could be established, one would have from this a Kuhn-Tucker characterization of the solutions of $\mathbf{P}$ : under some general hypothesis, $x \in X$ gives the minimum in $\mathbf{P}$ if and only if there is a $y \in Y$ such that $(x, y)$ is a saddle point of $L$. (And then $y$ gives the maximum in D.) While such a duality theorem can be developed, although under more restrictive assumptions than might be imagined, special techniques, involving the analysis of so-called induced constraints, are required. This development will not 
be pursued in the present paper. Here we establish instead a duality theorem of the type $\min \mathbf{P}=\sup \mathbf{D}$ and study solutions to $\mathbf{D}$ in terms of the perturbation function

$$
\varphi(u)=\inf _{x \in X} F(x, u), \quad u \in U
$$

The context is the case where the sets $C_{1}$ and $C_{2}$ are bounded, a property which is harmless to assume for most practical purposes, such as computation. Besides characterizing the directional derivatives to $\varphi$ at $u=0$ in terms of maximizing sequences to $\mathbf{D}$, we obtain results of a qualitative nature about the existence of solutions to $\mathbf{D}$. It is shown for instance that "typically" in this context $\mathbf{D}$ may be expected to have a solution $y$, so that the relation $\min \mathbf{P}=\max \mathbf{D}$ holds and the Kuhn-Tucker characterization of solutions $x$ to $\mathbf{P}$ mentioned above is valid.

The results of this paper complement those obtained for linear stochastic programs with recourse, i.e. when the functions $f_{1 k}$ and $f_{2 k}$ are affine in $x_{1}, x_{2}$ and $C_{1}, C_{2}$ are polytopes. For linear programs, a first pair of dual programs appear in [14] for which an inf = sup theorem was derived by imposing integrability restrictions on the random variables of the problems. (Earlier results of Madansky [5] have now been shown to be invalid [3].) In [2] Eisner and Olsen extend the results of Wets [14] to multistage problems as well as to other variants of the "stochastic program with recourse" model. It is noteworthy that these "dual" pairs are not cast as here in the $\mathscr{L}^{x}-\mathscr{L}^{1}$ framework but rather in the $\mathscr{L}^{p}-\mathscr{L}^{q}$ framework. This results from the rather specific form of constraints which allows one to show - under rather mild restrictions - that if $\mathbf{P}$ has an optimal solution then there exists in fact an optimal solution with $X$ replaced by $R^{n_{1}} \times \mathscr{L}_{n_{2}}^{p}$. The natural pairing is then $\mathscr{L}^{p}-\mathscr{L}^{q}$, see e.g. [14, Théorème 1 and Remarque 3]. Obviously these inf = sup theorems can be easily converted to $\mathrm{min}=$ sup if it is assumed that the constraints determine bounded regions for $x_{1}$ and $x_{2}$.

The following derivation of $\mathbf{D}$ for the linear case is useful in clarifying the relations between the results of this paper and those of [2] and [14]. Let $f_{10}\left(x_{1}\right)=c_{1} \times x_{1} ; f_{20}\left(s, x_{1}, x_{2}\right)=x_{2}(s) x_{2}, f_{1 k}\left(x_{1}\right)=b_{k}-A_{k}^{\prime} x_{1}$, $k=1, \cdots, m_{1} ; f_{2 k}\left(s, x_{1}, x_{2}\right)=p_{k}^{\prime}(s)-T_{k}^{\prime}(s) x_{1}-W_{k}^{\prime}(s) x_{2}$,

$$
C_{1}=\left\{x_{1} \mid b_{k}^{\prime \prime}-A_{k}^{\prime \prime} x_{1} \leqq 0, k=1, \cdots, \overline{m_{1}}, x_{1} \geqq 0\right\}
$$

and

$$
C_{2}=\left\{x_{2} \mid p^{\prime \prime}-W_{k}^{\prime \prime} x_{2} \leqq 0, k=1, \cdots, \overline{m_{2}}, x_{2} \geqq 0\right\}
$$


The sets $C_{1}, C_{2}$ are polytopes (bounded polyhedra). $E_{k}$ denotes the $k$ th row of the matrix $E$ and $E_{k}(s)$ indicates that the elements are random variables. Problem $\mathbf{P}$ then reads

$$
\text { Find }\left(x_{1}, x_{2}(s)\right) \in R^{n_{1}} \times \mathscr{L}_{n_{2}}^{\infty}
$$

satisfying

$$
A^{\prime \prime} x_{1} \geqq b^{\prime \prime}, x_{1} \geqq 0 \quad \text { and } \quad b^{\prime}-A^{\prime} x_{1} \leqq 0
$$

and almost surely

$$
W^{\prime \prime} x_{2}(s) \geqq p^{\prime \prime}, x_{2}(s) \geqq 0 \quad \text { and } \quad p^{\prime}(s)-T^{\prime}(s) x_{1}-W^{\prime}(s) x_{2}(s) \leqq 0 \text {, }
$$

and such that

$$
c_{1} x_{1}+\int_{s} c_{2}(s) x_{2}(s) \sigma(d s)
$$

is minimized. The Lagrangian becomes

$$
\begin{aligned}
& L(x, y)=c_{1} x_{1}+y_{1}\left(b^{\prime}-A^{\prime} x_{1}\right) \\
& \quad+\int_{s}\left[c_{2}(s) x_{2}(s)+y_{2}(s)\left(p^{\prime}(s)-T^{\prime}(s) x_{1}-W^{\prime}(s) x_{2}(s)\right)\right] \sigma(d s) \\
& \quad \text { if }\left\{\begin{array}{l}
\text { (i) } A^{\prime \prime} x_{1} \geqq b^{\prime \prime}, x_{1} \geqq 0 \text { and almost surely } W^{\prime \prime} x_{2}(s) \geqq p^{\prime \prime}, x_{2}(s) \geqq 0, \\
\text { (ii) } y_{1} \geqq 0 \text { and almost surely } y_{2}(s) \geqq 0 ; \\
=+\infty \text { if } x=\left(x_{1}, x_{2}(s)\right) \text { satisfies (i) but } y\left(y_{1}, y_{2}(s)\right) \text { does not satisfy (ii); } \\
=-\infty \text { if } y \text { does not satisfy (ii). }
\end{array}\right.
\end{aligned}
$$

The associated dual program $\mathbf{D}$ is obtained by maximizing $g(y)=$ $\operatorname{Inf}_{x \in X} L(x, y)$ on $y=R^{m_{1}} \times \mathscr{L}_{m_{2}}^{1}$. If there is no $x$ that satisfies (i) then $g(y) \equiv+\infty$ for all $y \in Y$. On the other hand if there is some $x$ satisfying (i) then $g(y)=-\infty$ for all $y$ that fails to satisfy (ii). Finally it remains to consider the case when the constraints of (i) are consistent and $y\left(y_{1}, y_{2}(s)\right)$ satisfies (ii). Observe that in this case $g$ can be written as

$$
\begin{aligned}
g(y)= & \operatorname{Min}_{\substack{A^{\prime \prime} x_{1} \geq b^{\prime \prime} \\
x_{1} \geq 0}}\left[c_{1}-y_{1} A^{\prime}-\int_{s} y_{2}(s) T^{\prime}(s) \sigma(d s)\right] x_{1} \\
& +\operatorname{Inf}_{\substack{W^{\prime \prime} x_{2}(s) \geq p^{\prime \prime} \text { a.s. } \\
x_{2}(s) \geqq 0 \text { a.s }}} \int_{s}\left[c_{2}(s)-y_{2}(s) W^{\prime}(s)\right] x_{2}(s) \sigma(d s)+y_{1} b \\
& +\int_{s} y_{2}(s) p^{\prime}(s) \sigma(d s)
\end{aligned}
$$


where a.s. stands for almost surely and min has replaced inf in the first term since $C_{1}=\left\{x_{1} \mid A^{\prime \prime} x_{1} \geqq b^{\prime \prime}, x_{1} \geqq 0\right\}$ is compact. We have written $g(y)$ in this form to stress that fact that the minimization of $L$ in $x$ can be done separately in $x_{1}$ and $x_{2}(s)$. Let $g_{1}(y)$ be the first term in the sum determining $g(y), g_{2}(y)$ the second and $g_{3}(y)$ the sum of the two last terms. For fixed $y, g_{3}(y)$ is a constant. The term $g_{1}(y)$ is just the optimal value of a linear program whose constraint set is nonempty and bounded; thus there are nonnegative Lagrange multipliers, say $\bar{y}_{1 k}$, $k=1, \cdots, \overline{m_{1}}$ such that

$$
g_{1}(y)=\operatorname{Min}_{x_{1} \geq 0}\left[c_{1}-y_{1} A^{\prime}-\bar{y}_{1} A^{\prime \prime}-\int_{s} y_{2}(s) T^{\prime}(s) \sigma(d s)\right] x_{1}+\bar{y}_{1} b^{\prime \prime} .
$$

Now $g_{1}(y)$ and consequently $g(y)$ will be $-\infty$ unless the vector $c_{1}-$ $y_{1} A^{\prime}-\bar{y}_{1} A^{\prime \prime}-\int_{S} y_{2}(s) T^{\prime}(s) \sigma(d s)$ is nonnegative. If this holds it follows that $g_{1}(y)=\bar{y}_{1} b$. Observe that the above conditions yield a complementary slackness condition. The term $g_{2}(y)$ can be handled similarly. Let $\bar{y}_{2 h}(s), k=1, \cdots, m_{2}$ be nonnegative Lagrange multipliers associated with the constraints $W^{\prime \prime} x_{2}(s) \geqq p^{\prime \prime}$ a.s. Assuming - as can be proved that $y_{2}(s)$ and $\bar{y}_{2}(s)$ can be selected measurable, we have that

$$
\begin{aligned}
g_{2}(y)=\operatorname{Inf}_{x_{2}(s) \geq 01} \int_{d . S}\left[c_{2}(s)-y_{2}(s) W^{\prime}(s)-\bar{y}_{2}(s) W^{\prime \prime}(s)\right] x_{2}(s) \sigma(d s) & \\
& +\int_{S} \bar{y}_{2}(s) p^{\prime \prime} \sigma(d s) .
\end{aligned}
$$

Again $g_{2}(y)$ will be $-\infty$ unless almost surely

$$
c_{2}(s)-y_{2}(s) W^{\prime}(s)-\bar{y}_{2}(s) W^{\prime \prime} \geqq 0,
$$

in which case $g_{2}(y)=\int_{S} \bar{y}_{2}(s) p^{\prime \prime} \sigma(d s)$. Regrouping all these conditions, we get the following form for $\mathbf{D}$ : Find $(\nu, \pi) \in R^{m_{1}+\bar{m}_{1}} \times \mathscr{L}_{m_{2}+\bar{m}_{2}}^{1}$ satisfying

$$
c_{1}-\nu A-\int_{S} \pi(s) T(s) \sigma(d s) \geqq 0, \nu \geqq 0
$$

and almost surely

$$
c_{2}(s)-\pi(s) W(s) \geqq 0, \pi(s) \geqq 0
$$

and such that 


$$
\nu b+\int_{s} \pi(s) p(s) \sigma(d s)
$$

is maximized (provided that $X_{0}$ be nonempty). We have identified the following symbols $\nu=\left(y_{1}, \bar{y}_{1}\right), \pi(s)=\left(y_{2}(s), \bar{y}_{2}(s)\right) \quad b=\left(\begin{array}{l}b^{\prime} \\ b^{\prime \prime}\end{array}\right) \quad p(s)=$ $\left(\begin{array}{c}p^{\prime}(s) \\ p^{\prime \prime}\end{array}\right) A=\left(\begin{array}{c}A^{\prime} \\ A^{\prime \prime}\end{array}\right), T(s)=\left(\begin{array}{c}T^{\prime}(s) \\ 0\end{array}\right), W(s)=\left(\begin{array}{c}W^{\prime}(s) \\ W^{\prime \prime}\end{array}\right)$ where 0 denotes the zero matrix of size $\bar{m}_{2} \times n_{1}$. The objective is obtained by collecting the remaining terms in $g_{1}, g_{2}$ and $g_{3}$. It is easy to compare the form of $\mathbf{D}$ to that of the "dual problem" of [14] which it resembles at least formally. The most obvious differences are that $W(s)$ is allowed to be random and that the second set of constraints have only to be satisfied almost surely. A hidden but significant difference is the compactness assumption on the constraint set of $\mathbf{P}$.

\section{Representation of the problem by an integral} functional. It is important for our purposes that the function $F$ defined above can also be expressed by

$$
F(x, u)=F_{1}\left(x_{1}, u_{1}\right)+\int_{s} F_{2}\left(s, x_{1}, x_{2}(s), u_{2}(s)\right) \sigma(d s),
$$

where

$$
\begin{aligned}
F_{1}\left(x, u_{1}\right) & =f_{10} \text { if } x_{1} \in C_{1} \text { and } f_{1 i}\left(x_{1}\right) \leqq u_{1 i}, \quad i=1, \cdots, m_{1} \\
& =+\infty \text { otherwise. }
\end{aligned}
$$

$$
\begin{aligned}
F_{2}\left(s, x_{1}, x_{2}, u_{2}\right) & =f_{20}\left(s, x_{1}, x_{2}\right) \text { if } x_{2} \in C_{2} \text { and } \begin{array}{r}
f_{2 i}\left(s, x_{1}, x_{2}\right) \leqq \\
i=1, \cdots, m_{2},
\end{array} \\
& =+\infty \text { otherwise. }
\end{aligned}
$$

Certain results will be obtained by applying the theory of convex integral functionals to the integrand $F_{2}$. The background is developed in this section.

As in [9], [10], [11], we shall call a function $h$ on $S \times R^{n}$ a normal convex integrand if $h(s, \cdot)$ is for each $s \in S$ a lower semicontinuous convex function on $R^{n}$ with values in $(-\infty,+\infty)$, not identically $+\infty$, and if furthermore there exists a sequence of measurable functions

$$
z^{k}: S \rightarrow R^{n}, \quad k=1,2, \cdots,
$$

such that $h\left(s, z^{k}(s)\right)$ is measurable in $s$ for each $k$, while for each fixed $s$ the set of points of the form $z^{k}(s)$ lying in 


$$
\operatorname{dom} h(s, \cdot)=\left\{z \in R^{n} \mid h(s, z)<+\infty\right\}
$$

are dense in the latter set. Various criteria for normality are given in [9], [10], [11].

This property implies in particular that $h(s, z(s))$ is measurable in $s$ when $z(s)$ is measurable in [10, Corollary of Lemma 5]. The integral functional

$$
I_{h}(z)=\int_{s} h(s, z(s)) \sigma(d s)
$$

therefore has a well-defined value (real or $-\infty$ ) for every measurable function $z$ such that $h(s, z(s))$ is majorized by a summable function of $s$; when $h(s, z(s))$ is not so majorized, we adopt the convention that $I_{h}(z)=+\infty$. This convention is used more generally below to give a meaning to $\int_{S} \theta(s) \sigma(d s)$ for any measurable, extended-real-valued function $\theta$.

The following fact was essentially proved in [10, Theorem 4].

Proposition 1. Let $h$ be a normal convex integrand on $S \times$ $R^{n}$. Then the infimum of $h(s, \cdot)$ over $R^{n}$ is measurable as a function of $s$, and one has

$$
\inf _{z \in \mathscr{L}_{n}^{p}} \int_{S} h(s, z(s)) \sigma(d s)=\int_{S}\left[\inf _{z \in R^{n}} h(s, z)\right] \sigma(d s)
$$

for any $p \in[1,+\infty]$ such that the infimum on the left is not $+\infty$.

Proof. The normality of $h$ implies that the conjugate integrand

$$
h^{*}(s, w)=\sup _{z \in R^{n}}\{z \cdot w-h(s, z)\}
$$

is likewise normal [10, Lemma 5] and hence in particular is measurable in $s$ for fixed $w$. Since

$$
-h^{*}(s, 0)=\inf _{z \in R^{n}} h(s, z),
$$

we conclude that the latter infimum is measurable as a function of $s$.

It is obvious that the inequality $\geqq$ is valid in (2.5). It suffices to show therefore that, if $\alpha: S \rightarrow R$ is any summable function satisfying

$$
\inf _{z \in R^{n}} h(s, z) \leqq \alpha(s),
$$


then

$$
\inf _{z \in \mathscr{L}_{n}^{p}} \int_{S} h(s, z(s)) \sigma(d s) \leqq \int_{s} \alpha(s) \sigma(d s)
$$

Define

$$
r(s, z)=\max \{h(s, z), \alpha(s)\}
$$

Then $r$ is another normal convex integrand (immediate from the definition; see also [9, Cor. 4.1]). It will be enough to show

$$
\inf _{z \in \mathscr{L}_{n}^{p}} \int_{S} r(s, z(s)) \sigma(d s)=\int_{S} \alpha(s) \sigma(d s) .
$$

Arguing as above, we use the fact that the conjugate integrand

$$
r^{*}(s, w)=\sup _{z \in R^{n}}\{z \cdot w-r(s, z)\}
$$

is also normal and satisfies

$$
-r^{*}(s, 0)=\inf _{z \in R^{n}} r(s, z)=\alpha(s) .
$$

Thus the integral functional $I_{r^{*}}$ on $\mathscr{L}_{n}^{q}($ where $1 / p+1 / q=1)$ is finite at the origin, while on the other hand, since $I_{h}$ is not identically $+\infty$ on $\mathscr{L}_{n}^{p}$, the integral functional $I_{r}$ is finite at some point of $\mathscr{L}_{n}^{p}$. It follows then from a basic theorem on integral functionals [10, Theorem 2] that $I_{r}$ and $I_{r^{*}}$ are conjugate to each other with respect to the natural pairing between $\mathscr{L}_{n}^{p}$ and $\mathscr{L}_{n}^{q}$, and hence in particular

$$
I_{r^{*}}(0)=\sup _{z \in \mathscr{L}_{n}^{p}}\left\{\langle z, 0\rangle-I_{r}(z)\right\}=-\inf _{z \in \mathscr{L}_{n}^{p}} I_{r}(z)
$$

Recalling (2.9), one sees that (2.10) is precisely the desired relation (2.8).

Proposition 2. The function $F_{2}$ is a normal convex integrand on $S \times R^{n_{1}} \times R^{n_{2}} \times R^{m_{2}}$.

Proof. Let

$$
\begin{aligned}
& F_{21}\left(s, x_{1}, x_{2}, u_{2}\right)=f_{2 i}\left(s, x_{1}, x_{2}\right)-u_{2 i} \text { for } i=1, \cdots, m_{2}, \\
& F_{20}\left(s, x_{1}, x_{2}, u_{2}\right)=f_{20}\left(s,{ }^{\prime} x_{1}, x_{2}\right) .
\end{aligned}
$$


Then $F_{2 l}\left(s, x_{1}, x_{2}, u_{2}\right)$ is finite, measurable in $s$ and convex in $\left(x_{1}, x_{2}, u_{2}\right)$, so that $F_{2 l}$ is a normal convex integrand, $i=0,1, \cdots, m_{2}[\mathbf{4}$, Lemma 2]. For $i=1, \cdots, m_{2}$ let

$$
\begin{aligned}
h_{\iota}\left(s, x_{1}, x_{2}, u_{2}\right) & =0 \quad \text { if } \quad F_{2 t}\left(s, x_{1}, x_{2}, u_{2}\right) \leqq 0 \\
& =+\infty \quad \text { if } \quad F_{2 i}\left(s, x_{1}, x_{2}, u_{2}\right)>0
\end{aligned}
$$

Then $h_{i}$ is a normal convex inegrand [9, Theorem 4, Cor. 4.4]. Furthermore the function

$$
\begin{aligned}
h\left(s, x_{1}, x_{2}, u_{2}\right) & =0 \quad \text { if } \quad x_{2} \in C_{2} \\
& =+\infty \quad \text { if } \quad x_{2} \notin C_{2}
\end{aligned}
$$

is a normal convex integrand [10, Lemma 1]. We have

$$
F_{2}=F_{20}+h+\sum_{i=1}^{m_{2}} h_{t}
$$

and $F_{2}(s, \cdot, \cdot, \cdot)$ is not identically $+\infty$ on $R^{n_{1}} \times R^{m_{2}} \times R^{m_{2}}$ for any s. Therefore, as a sum of normal convex integrands, $F_{2}$ is a normal convex integrand [9, Cor. 4.2].

The last result of this section involves continuity properties of $F$ as a functional on $X \times U$. Of course, $X \times U$ has a natural normable topology, but we shall also be interested in the weak topology which is the product of the weak topology on $U$ induced by the pairing (1.6) with the space $Y$ and the weak topology on $X$ induced by the pairing

$$
\langle x, v\rangle=x_{1} \cdot v_{1}+\int_{S} x_{2}(s) \cdot v_{2}(s) \sigma(d s)
$$

for

$$
v=\left(v_{1}, v_{2}\right) \in V=R^{n_{1}} \times \mathscr{L}_{n_{2}}^{1} .
$$

Proposition 3. The functional F on $X \times U$ is lower semicontinuous, convex and not identically $+\infty$, the lower semicontinuity being not only with respect to the normable topology, but also with respect to the weak topology on $X \times U$ induced by the above pairing with $V \times Y$.

Proof. It is easy to see that $F \not \equiv+x$ : choose any $\left(x_{1}, x_{2}\right)$ in $X_{0}$, the set defined in (1.9). Since the function $s \rightarrow\left(x_{1}, x_{2}(s)\right)$ is measurable and essentially bounded, we know that the functions $s \rightarrow f_{2 i}\left(s, x_{1}, x_{2}(s)\right)$ for $i=1, \cdots, m_{2}$ are measurable and essentially bounded. Define $u=$ $\left(u_{1}, u_{2}\right) \in U$ by 


$$
\begin{array}{ll}
u_{1 i}=f_{1 i}\left(x_{1}\right), & i=1, \cdots, m_{1}, \\
u_{2 i}(s)=f_{2 i}\left(s, x_{1}, x_{2}(s)\right), & i=1, \cdots, m_{2} .
\end{array}
$$

The constraints (1.4) and (1.5) are then satisfied, so $F(x, u)$ has by definition a finite value.

The convexity of $F$ is obvious from (2.1) and the convexity of the functions $F_{1}$ and $F_{2}(s ; \cdot, \cdot, \cdot)$. To establish the lower semicontinuity, it will be enough to show, setting

$$
R^{n}=R^{n_{1}} \times R^{m_{2}} \times R^{m_{2}}, \quad z=\left(x_{1}, x_{2}, u_{2}\right),
$$

that the integral functional

$$
I_{F_{2}}(z)=\int_{S} F_{2}(s, z(s)) \sigma(d s)
$$

is lower semicontinuous on $\mathscr{L}_{n}^{\infty}$ with respect to the weak topology induced by $\mathscr{L}_{n}^{1}$.

From what we have already noted, there is at least one $z \in \mathscr{L}_{n}^{\infty}$ such that $I_{F_{2}}(z)<+\infty$. We shall demonstrate for the conjugate integrand

$$
F_{2}^{*}(s, w)=\sup _{z \in R^{n}}\left\{z: w-F_{2}(s, z)\right\}
$$

it is also true that $I_{F_{2}^{*}}(w)<+\infty$ for some $w \in \mathscr{L}_{n}^{1}$. This will imply by the fundamental theorem on integral functionals invoked above in proving Proposition 1 [10, Theorem 2] that $I_{F_{2}}$ on $\mathscr{L}_{n}^{\infty}$ and $I_{F_{2}}$ on $\mathscr{L}_{n}^{1}$ are conjugate to each other with respect to the natural pairing between $\mathscr{L}_{n}^{\infty}$ and $\mathscr{L}_{n}^{1}$, and hence in particular are lower semicontinuous with respect to the weak topologies induced by this pairing.

Define $h$ on $S \times R^{n}$ by

$$
h(s, z)=f_{20}\left(s, x_{1}, x_{2}\right) \text { for } \quad z=\left(x_{1}, x_{2}, u_{2}\right) .
$$

Since $h$ is finite, convex in $z$ and summable in $s$, it is a normal convex integrand [10, Lemma 2]. Indeed, according to [11, Theorem 2], the integral functionals $I_{h}$ on $\mathscr{L}_{n}^{\infty}$ and $I_{h^{*}}$ on $\mathscr{L}_{n}^{1}$ are conjugate to each other. The latter relationship implies $I_{h^{*}}$ is not identically $+\infty$, since otherwise the conjugate of $I_{h}$ * would be identically $-\infty$, contrary to $I_{h}$ being finite on the constant functions (in fact finite throughout $\mathscr{L}_{n}^{\infty}$ ). Since $h \leqq F_{2}$, we have $h^{*} \geqq F_{2}^{*}$. Taking any $w \in \mathscr{L}_{n}^{1}$ such that $I_{h^{*}}(w)<+\infty$, we have $I_{F_{2}}(w)<+\infty$ as desired. 
3. The problem seen from the first stage. To provide some partial justification for our restriction to recourse functions which are measurable and essentially bounded, we consider the relationship between the problem in this formulation and two versions of the problem in terms of the initial choice of $x_{1}$.

For convenience, let us denote by $f$ the essential objective function in $\mathbf{P}$; thus $f(x)=F(x, 0)$ or in more complete fashion

$$
\begin{gathered}
f\left(x_{1}, x_{2}\right)=F_{1}\left(x_{1}, 0\right)+\int_{S} F_{2}\left(s, x_{1}, x_{2}(s), 0\right) \sigma(d s) \\
\text { for }\left(x_{1}, x_{2}\right) \in X=R^{n_{1}} \times \mathscr{L}_{n_{2}}^{\infty} .
\end{gathered}
$$

As seen fron the first stage, $\mathbf{P}$ amounts to minimizing the function

$$
J\left(x_{1}\right)=\inf _{x_{2} \in \mathscr{L}_{n_{2}}^{\infty}} f\left(x_{1}, x_{2}\right)
$$

over $R^{n_{1}}$; this may be termed the first-stage problem induced by $\mathbf{P}$. On the other hand, for each $x_{1} \in R^{n_{1}}$ and $s \in S$ let

$$
q\left(s, x_{1}\right)=\inf _{x_{2} \in R^{n_{2}}} F_{2}\left(s, x_{1}, x_{2}, 0\right)
$$

in other words, $q\left(s, x_{1}\right)$ is the infimum of the second-stage cost $f_{20}\left(s, x_{1}, x_{2}\right)$ over all recourses $x_{2}$ satisfying the second-stage constraints. The problem

$$
\mathbf{Q} \text { minimize } j\left(x_{1}\right)=F_{1}\left(x_{1}, 0\right)+\int_{S} q\left(s, x_{1}\right) \sigma(d s) \text { over all } x_{1} \in R^{n_{1}}
$$

may then be termed the intrinsic first-stage problem. Here, following our earlier convention, we regard $j\left(x_{1}\right)$ as $+\infty$ unless $F_{1}\left(x_{1}, 0\right)<+\infty$ and the function $s \rightarrow q\left(s, x_{1}\right)$ is majorized by a summable function of $s$. The measurability which is needed in this definition is asserted by the next proposition.

Proposition 4. For each $x_{1} \in R^{n_{1}}, q\left(s, x_{1}\right)$ is measurable as a function of $s \in S$.

Proof. Fix $x_{1}$, let $h\left(s, x_{2}\right)=F_{2}\left(s, x_{1}, x_{2}, 0\right)$ and

$$
S^{\prime}=\left\{s \in S \mid \exists x_{2} \text { with } h\left(s, x_{2}\right)<+\infty\right\}:
$$


Since $F_{2}$ is a normal convex integrand (Proposition 2), the set $S^{\prime}$ is measurable, and $h$ is a normal convex integrand on $S^{\prime} \times R^{n_{2}}[9$, Corollary 4.5]. We may conclude then from Proposition 1 and the formula

$$
q\left(s, x_{1}\right)=\inf _{x_{2} \in R^{n_{2}}} h\left(s, x_{2}\right)
$$

that $q\left(s, x_{1}\right)$ is measurable as a function of $s \in S^{\prime}$. Since $q\left(s, x_{1}\right)=+\infty$ for $s \notin S^{\prime}$, the result is thereby established.

Obviously it is true that

$$
j\left(x_{1}\right) \leqq f\left(x_{1}, x_{2}\right) \text { for all } x_{1} \in R^{n_{1}}, x_{2} \in \mathscr{L}_{n_{2}}^{\infty},
$$

and therefore

$$
\inf \mathbf{Q} \leqq \inf \mathbf{P} \text {. }
$$

The question in justifying $\mathbf{P}$ is whether under fairly general circumstances equality holds, and if so, whether solutions to one problem correspond to solutions to the other. More specifically, we may ask under what assumptions the function $J$ in (3.2) coincides with $j$, and when the infimum in the definition of $J$ is attained.

Let $\rho\left(s, x_{1}\right)$ denote the distance from the origin of the set of all $x_{2} \in R^{m_{2}}$ satisfying the second-stage constraints corresponding to $x_{1}$ and $s$ :

$$
\rho\left(s, x_{1}\right)=\inf \left\{\left|x_{2}\right| \mid F_{2}\left(s, x_{1}, x_{2}, 0\right)<+\infty\right\} .
$$

(By convention, $\rho\left(s, x_{1}\right)=+\infty$ if the set is empty.) We shall say that a point $x_{1}$ is intrinsically feasible in the first stage if $j\left(x_{1}\right)<+\infty$, which implies of course that $x_{1}$ satisfies the first-stage constraints (1.1) and has $\rho\left(s, x_{1}\right)<+\infty$ except for a set of probability measure zero.

THEOREM 1. Suppose that for each $x_{1}$ which is intrinsically feasible in the first stage $\rho\left(s, x_{1}\right)$ is essentially bounded in $s$. Then

$$
j\left(x_{1}\right)=\inf _{x_{2} \in \mathscr{L}_{n_{2}}^{\infty}} f\left(x_{1}, x_{2}\right) \quad \text { for all } \quad x_{1} \in R_{1}^{n}
$$

In particular one has inf $\mathbf{Q}=\inf \mathbf{P}$, and $x_{1}$ gives the minimum in the intrinsic first-stage problem $\mathbf{Q}$ if and only if it gives the minimum in the first-stage problem induced by $\mathbf{P}$.

Proof. Relation (3.8) is trivial if $x_{1}$ is not intrinsically feasible in the 
first stage, so we henceforth fix such an $x_{1}$. Consider the set $S^{\prime}$ and normal convex integrand $h$ on $S^{\prime} \times R^{m_{2}}$ defined in the proof of Proposition 4. Since (3.4) holds and $j\left(x_{1}\right)<+\infty, S^{\prime}$ differs from $S$ only by a set of zero measure. Thus (3.8) is equivalent to the relation

$$
\int_{S^{\prime}}\left[\inf _{x_{2} \in R^{n_{2}}} h\left(s, x_{2}\right)\right] \sigma(d s)=\inf _{x_{2} \in \mathscr{L}_{n_{2}}^{\infty}} \int_{s^{\prime}} h\left(s, x_{2}(s)\right) \sigma(d s),
$$

and this is valid by Proposition 1 if the infimum over $\mathscr{L}_{n_{2}}^{\infty}$ is not $+\infty$. We can therefore prove the theorem by showing the existence of $x_{2} \in \mathscr{L}_{n_{2}}^{\infty}$ with $F_{2}\left(s, x_{1}, x_{2}(s), 0\right)$ summable in $s$. Since $F_{2}$ is given by (2.3), where $f_{20}\left(s, x_{1}, x_{2}(s)\right)$ is always summable in $s$ for $x_{2} \in \mathscr{L}_{n_{2}}^{\infty}$, the equation reduces to the following. For each $s \in S$ let

$$
\Gamma(s)=\left\{x_{2} \in R^{m_{2}} \mid x_{2} \in C_{2} \quad \text { and } \quad f_{2 i}\left(s, x_{1}, x_{2}\right) \leqq 0, i=1, \cdots, m_{2}\right\} .
$$

We want $x_{2} \in \mathscr{L}_{n_{2}}^{\infty}$ satisfying $x_{2}(s) \in \Gamma(s)$ almost surely. Observe that the multifunction $\Gamma$ is closed-convex-valued, and $\Gamma(s) \neq \phi$ for $s \in S^{\prime}$. We claim $\Gamma$ is also measurable (in the sense that $\{s \mid \Gamma(s) \cap K \neq \phi\}$ is measurable in $S$ for every closed $k \subset R^{n_{2}}$ ). This follows from (3.8) and the fact that $C_{2}$ is closed and the functions

$$
h_{i}\left(s, x_{2}\right)=f_{2 i}\left(s, x_{1}, x_{2}\right), \quad i=1, \cdots, m_{2},
$$

being finite, measurable in $s$ and convex in $x_{2}$, are normal convex integrands; see [9, Corollary 4.4]. Because

$$
\rho\left(s, x_{1}\right)=\operatorname{dist}(0, \Gamma(s)),
$$

we may conclude $\rho\left(s, x_{1}\right)$ is measurable in $s$; in fact, if $x_{2}(s)$ denotes for each $s \in S^{\prime}$ the unique point of $\Gamma(s)$ nearest 0 , then $x_{2}$ is a measurable function on $S^{\prime}\left[9\right.$, Theorem 3(f)], and of course $\left|x_{2}(s)\right|=\rho\left(s, x_{1}\right)$. Since $\rho\left(s, x_{1}\right)$ is essentially bounded in $s$ by hypothesis, and $S^{\prime}$ differs from $S$ only by a set of measure 0 , the function $x_{2}$ so constructed may be regarded as an element of $\mathscr{L}_{n_{2}}^{\infty}$ satisfying $x_{2}(s) \in \Gamma(s)$ almost surely, as desired.

THEOREM 2. Suppose the set $C_{2}$ is bounded. Then not only are the conclusions of Theorem 1 valid, but also the infimum in (3.8) is attained for each $x_{1} \in R^{n_{1}}$ by at least one $x_{2} \in \mathscr{L}_{n_{2}}^{\infty}$.

Proof. The hypothesis of Theorem 1 is satisfied, becuase

$$
\rho\left(s, x_{1}\right) \leqq r=\max \left\{\left|x_{2}\right| \mid x_{2} \in C_{2}\right\} .
$$


Attainment in (3.8) is trivial if $j\left(x_{1}\right)=+\infty$, so fix $x_{1}$ with $j\left(x_{1}\right)<+\infty$ and consider the set $S^{\prime}$ and normal convex integrand $h$ on $S^{\prime} \times R^{n}$ defined in the proof of Proposition 4. As observed in the proof of Theorem 1, $S^{\prime}$ differs from $S$ only by a set of measure zero, and (3.8) can be written as (3.9). The question reduces thus to whether the infimum on the right in (3.9) is attained. For each $s \in S^{\prime}$, let $\Gamma(s)$ denote the set of all $x_{2} \in R^{n_{2}}$ at which the infimum of $h(s, \cdot)$ is attained. Since $h\left(s,{ }^{\cdot}\right)$ is lower semicontinuous and

$$
h\left(s, x_{2}\right)<+\infty \text { implies } x_{2} \in C_{2} \text {, }
$$

$\Gamma(s)$ is a nonempty, compact subset of $C_{2}$. Moreover $\Gamma$ is a measurable multifunction from $S^{\prime}$ to $R^{n_{2}}$, because $h$ is a normal convex integrand [9, Theorem 4]. There exists therefore a measurable function $x_{2}: S^{\prime} \rightarrow R^{n_{2}}$ such that $x_{2}(s) \in \Gamma(s)$ for every $s$ [9, Corollary 1.1]. This function satisfies in particular $x_{2}(s) \in C_{2}$ and hence is bounded. Thus it can be identified with an element of $\mathscr{L}_{n_{2}}^{\infty}$ for which the infimum on the right in (3.9) is attained.

Corollary. Suppose the set $C_{2}$ is bounded. Then $x_{1} \in R^{n_{1}}$ gives the minimum in $\mathbf{Q}$ if and only if there exists $x_{2} \in \mathscr{L}_{n_{2}}^{\infty}$ such that $x=\left(x_{1}, x_{2}\right)$ gives the minimum in $\mathbf{P}$.

If the functions $f_{1 k}, f_{2 k}$ are linear and $C_{1}, C_{2}$ are convex polyhedral (not necessarily bounded) one can then use the somewhat more specialized results of linear parametric programming to obtain variants of Theorems 1 and 2, see [4], [14] and [6].

4. Duality. The properties of the functional $F$ established in Proposition 3 allow us to apply the general duality theory of [8] to $\mathbf{P}$ and D. Thus the perturbation function $\varphi$ defined in (1.17) is convex on $U$, and its conjugate $\varphi^{*}$ on $Y$ with respect to the pairing (1.6) is related to the concave essential objective function $g$ in $\mathbf{D}$ by

$$
g(y)=\inf _{u \in U}\{\langle u, y\rangle+\varphi(u)\}=-\varphi^{*}(-y) .
$$

In terms of the biconjugate $\varphi^{* *}$, one has

$$
\varphi^{* *}(0)=\sup \mathbf{D}
$$

whereas of course

$$
\varphi(0)=\inf \mathbf{P}
$$


Moreover

$$
\varphi^{* *}(0)=\liminf _{u \rightarrow 0} \varphi(u)
$$

if for instance $\varphi^{* *}(0)>-\infty$, or if the limit in (4.4) is not $+\infty$, the limit is taken with respect to the weak topology induced on $U$ by $Y$. The relation

$$
g(y)=\max \mathbf{D}=\inf \mathbf{P}
$$

is equivalent to the subgradient relation

$$
-y \in \partial \varphi(0)
$$

or in other words

$$
\varphi(u) \geqq \varphi(0)-\langle u, y\rangle \text { for all } u \in U .
$$

This can be interpreted in turn as saying that $y$ is an "equilibrium price system" for $\mathbf{P}$, in the sense that the expression

$$
F(x, u)+\langle u, y\rangle
$$

is minimized over $X \times U$ when $u=0$ and $x$ gives the minimum in $\mathbf{P}$. The duality theorem below leads to a sharpening of these facts.

THEOREM 3. Suppose the sets $C_{1}$ and $C_{2}$ are bounded. Then

$$
\min \mathbf{P}=\sup \mathbf{D}>-\infty
$$

In fact, the perturbation function $\varphi$ is a proper convex function on $U$ which is lower semicontinuous with respect to the weak topology on $U$ induced by the pairing with $Y$, and the infimum defining $\varphi$ is always attained, i.e. for each $u \in U$ there exists at least one $x \in X$ such that $F(x, u)=\varphi(u)$. In particular, $\varphi^{* *}=\varphi$.

Proof. The relation $\min \mathbf{P}=\sup \mathbf{D}$ follows from (4.2), (4.3), and the asserted properties of $\varphi$. To derive these properties, we could invoke broader results of Rockafellar [8] or Wets [13], but it is easy in the case at hand to furnish a direct proof.

First we argue that the set $X_{0} \subset X$, defined in (1.9), is under the present hypothesis compact relative to the weak topology induced on $X$ by $V$ in the pairing $(2.11),(2.12)$. This amounts to the assertion that the set 


$$
X_{0}^{\prime}=\left\{x_{2} \in \mathscr{L}_{n_{2}}^{\infty} \mid x_{2}(s) \in C_{2} \text { almost surely }\right\}
$$

is compact in the weak topology induced on $\mathscr{L}_{n_{2}}^{\infty}$ by $\mathscr{L}_{n_{2}}^{1}$. Certainly $X_{0}^{\prime}$ is relatively compact in this topology, inasmuch as $C_{2}$ is bounded. We verify that $X_{0}^{\prime}$ is also closed, hence indeed compact, by considering the function $h$ on $S \times R^{m_{2}}$ defined by

$$
\begin{aligned}
h\left(s, x_{2}\right) & =0 \quad \text { if } \quad x_{2} \in C_{2}, \\
& =+\infty \quad \text { if } \quad x_{2} \notin C_{2} .
\end{aligned}
$$

This is a normal convex integrand, because $C_{2}$ is a nonempty, closed, convex set [10, Lemma 1]. The corresponding integral functional $I_{h}$ on $\mathscr{L}_{n_{2}}^{\infty}$ satisfies

$$
\begin{aligned}
I_{h}\left(x_{2}\right) & =0 \quad \text { if } \quad x_{2} \in X_{0}^{\prime}, \\
& =+\infty \quad \text { if } \quad x_{2} \notin X_{0}^{\prime} .
\end{aligned}
$$

In particular, $I_{h}\left(x_{2}\right)<+\infty$ for at least one $x_{2} \in \mathscr{L}_{n_{2}}^{\infty}$. On the other hand, the conjugate integrand

$$
h^{*}\left(s, v_{2}\right)=\sup _{x_{2} \in R^{n_{2}}}\left\{x_{2} \cdot v_{2}-h\left(s, x_{2}\right)\right\}
$$

has $h^{*}(s, 0) \equiv 0$, and hence $I_{h^{*}}\left(v_{2}\right)<+\infty$ for at least one $v_{2} \in \mathscr{L}_{n_{2}}^{1}$, namely $v_{2}=0$. It follows that $I_{h}$ on $\mathscr{L}_{n_{2}}^{\infty}$ and $I_{h^{*}}$ on $\mathscr{L}_{n_{2}}^{1}$ are convex functionals conjugate to each other [10, Theorem 2], and this implies, among other things, that $I_{h}$ is lower semicontinuous with respect to the weak topology induced on $\mathscr{L}_{n_{2}}^{\infty}$ by $\mathscr{L}_{n_{2}}^{1}$. But

$$
X_{0}^{\prime}=\left\{x_{2} \in \mathscr{L}_{n_{2}}^{\infty} \mid I_{h}(x) \leqq 0\right\},
$$

in view of (4.11). Therefore $X_{0}^{\prime}$ is closed as claimed.

The compactness of $X_{0}$ implies that in the definition

$$
\varphi(u)=\inf _{x \in X} F(x, u)
$$

the infimum is always attained, since $F$ is lower semicontinuous in the weak topology (Proposition 3), and

$$
F(x, u)<+\infty \text { implies } x \in X_{0} .
$$

Thus, like $F$ (Proposition 3), $\varphi$ is not identically $+\infty$ and nowhere has the 
value $-\infty$ (i.e. $\varphi$ is "proper"), and each of the level sets $\{u \in U \mid \varphi(u)$ $\leqq \alpha\}$ is the projection on $U$ of the corresponding level set of $F$ :

$$
D_{\alpha}=\{(x, u) \in X \times U \mid F(x, u) \leqq \alpha\} .
$$

But the projection of $D_{\alpha}$ on $U$ is closed in the weak topology. This is true because $D_{\alpha}$ is closed (by the lower semicontinuity of $F$ ), and the projection of $D_{\alpha}$ on $X$ is contained in the compact set $X_{0}$. Therefore $\varphi$ is lower semicontinuous (in the weak topology induced on $U$ by $Y$ ). Inasmuch as $\varphi$ is a proper convex function on $U$ which is lower semicontinuous in a topology compatible with the pairing between $U$ and $Y$, we have $\varphi^{* *}=\varphi$.

Corollary 1. Suppose the sets $C_{1}$ and $C_{2}$ are bounded. Then $y$ gives the maximum in $\mathbf{D}$ if and only if $-y \in \partial \varphi(0)$. Thus if $\partial \varphi(0) \neq \varnothing$ one has the following characterization: $x \in X$ gives the minimum in $\mathbf{P}$ if and only if there exists $y \in Y$ such that $(x, y)$ is a saddle point of the Lagrangian $L$.

The interesting feature of this corollary is the qualitative information it provides about the necessity of the saddle point criterion for optimality, in reducing this necessity to the question of whether $\partial \varphi(0) \neq \varnothing$. Let us think of $\mathbf{P}$ again as embedded in the class of perturbed problems $\mathbf{P}(u)$. If $\mathbf{P}$ is replaced by a particular $\mathbf{P}(u)$, this amounts merely to a translation of $F$ in the space $U$; the Lagrangian $L$ is replaced correspondingly by

$$
L_{u}(x, y)=L(x, y)-\langle u, y\rangle
$$

and the dual $\mathbf{D}$ is replaced by

$$
\mathbf{D}(u) \text { : maximize } g(y)-\langle u, y\rangle \text { over all } y \in Y \text {. }
$$

Assuming the sets $C_{1}$ and $C_{2}$ are bounded, we have

$$
\min \mathbf{P}(u)=\sup \mathbf{D}(u),
$$

where the supremum in $\mathbf{D}(u)$ is attained at $y$ if and only if $-y \in$ $\partial \varphi(u)$. Let us say that $\mathbf{P}(u)$ is a regular problem if

$$
\min \mathbf{P}(u)=\max \mathbf{D}(u),
$$

so that the saddle point criterion for optimality in $\mathbf{P}(u)$, in terms of the Lagrangian $L_{u}$, is a necessary condition. We then have: 
Corollary 2. Suppose the sets $C_{1}$ and $C_{2}$ are bounded. Then $\mathbf{P}(u)$ is a regular problem for every $u$ such that $\partial \varphi(u) \neq \varnothing$. Moreover, given any $u \in U$ such that inf $\mathbf{P}(u)<+\infty$, and any $y \in Y$ and $\epsilon>0$ such that $y$ is an $\epsilon$-solution to the dual $\mathbf{D}(u)$ (i.e. yields the maximum in $\mathbf{D}(u)$ to within $\epsilon$ ), there exist $u^{\prime} \in U$ and $y^{\prime} \in Y$ with

$$
\left\|u^{\prime}-u\right\|_{\infty} \leqq \sqrt{\epsilon} \text { and }\left\|y^{\prime}-y\right\|_{1} \leqq \sqrt{\epsilon}
$$

such that $\mathbf{P}\left(u^{\prime}\right)$ is a regular problem and $y^{\prime}$ gives the maximum in $\mathbf{D}\left(u^{\prime}\right)$.

The norms here are

$$
\begin{aligned}
& \|y\|_{1}=\left|y_{1}\right|+\left\|y_{2}\right\|_{1} \quad \text { for } \quad y=\left(y_{1}, y_{2}\right) \in Y, \\
& \|u\|_{\infty}=\max \left\{\left|u_{1}\right|,\left\|u_{2}\right\|_{\infty}\right\} \quad \text { for } \quad u=\left(u_{1}, u_{2}\right) \in U .
\end{aligned}
$$

The last part of the corollary is a restatement of an existence lemma for subgradients in Brøndsted-Rockafellar [1]: if $-y \in \partial_{\epsilon} \varphi(u)$, then there exist $u^{\prime}$ and $y^{\prime}$ satisfying (4.18) such that $-y^{\prime} \in \partial \varphi\left(u^{\prime}\right)$. This is applicable because $Y$ is a Banach space under the norm (4.19) whose dual can be identified with $U$ under the norm (4.20), and because $\varphi$ is according to Theorem 1 the conjugate of a convex function on $Y$ (namely $\varphi^{*}$, since $\left.\varphi^{* *}=\varphi\right)$. The elements $y$ such that $-y$ belongs to the $\epsilon$-subgradient set $\partial_{\epsilon} \varphi(u)$ are the $\epsilon$-solutions to $\mathbf{D}(u)$, in view of (4.1). We have seen, on the other hand, that the relation $-y^{\prime} \in \partial \varphi\left(u^{\prime}\right)$ means that $\mathbf{P}\left(u^{\prime}\right)$ is a regular problem and $y^{\prime}$ gives the maximum in $\mathbf{D}\left(u^{\prime}\right)$.

In particular, Corollary 2 says that the set of $u$ such that $\mathbf{P}(u)$ is a regular problem is norm-dense in the set of $u$ such that inf $\mathbf{P}(u)<+\infty$.

The final result of this paper furnishes a complete characterization of the directional derivatives

$$
\varphi^{\prime}(0, u)=\lim _{\lambda \downarrow 0}\{\varphi(\lambda u)-\varphi(0)] / \lambda .
$$

These exist of course by the convexity of $\varphi$, when $\varphi(0)$ is finite.

Theorem 4. Suppose the sets $C_{1}$ and $C_{2}$ are bounded, and inf $\mathbf{P}<$ $+\infty$. Then for every $u \in U$ one has

$$
\varphi^{\prime}(0 ; u)=\lim _{\epsilon \downarrow 0} \sup _{y \in M(\epsilon)}\langle u,-y\rangle,
$$

where $M(\epsilon)$ is the set of $\epsilon$-solutions to $\mathbf{D}$. 
Proof. The hypothesis implies by Theorem 3 that $\varphi(0)$ is finite and $\varphi^{* *}=\varphi$. The formula

$$
\varphi^{\prime}(0 ; u)=\lim _{\epsilon \downarrow 0} \sup _{y \in \partial_{\epsilon \varphi}(0)}\langle u, y\rangle
$$

is then valid [8, Theorem 3]. The $\epsilon$-subgradient set $\partial_{\epsilon} \varphi(0)$, consisting of the elements $y$ such that

$$
\varphi(u) \geqq[\varphi(0)-\epsilon]+\langle u, y\rangle \text { for all } u \in U,
$$

coincides with $-M(\epsilon)$ by (4.1).

Corollary. Suppose the sets $C_{1}$ and $C_{2}$ are bounded, and inf $\mathbf{P}<$ $+\infty$. Let $u \in U$ and $\alpha \in R$. Then the inequality $\varphi^{\prime}(0, u) \geqq \alpha$ holds if and only if there exists a maximizing sequence $\left(y^{k}\right)_{k=1}^{\infty}$ for D such that

$$
\limsup _{k \rightarrow \infty}\left\langle u,-y^{k}\right\rangle \geqq \alpha
$$

5. An example. Here we give a very simple example which shows that this theory allows only for a $\min =$ sup duality theorem. Let us consider the following problem P: $\operatorname{Find}\left(x_{1}, x_{2}(s)\right) \in R^{n_{1}} \times \mathscr{L}_{n_{2}}^{\infty}$ satisfying

$$
x_{1} \in C_{1}=\left\{x_{1} \mid 0 \leqq x_{1} \leqq 1\right\}
$$

and almost surely

$$
x_{2} \in C_{2}=\left\{x_{2} \mid 0 \leqq x_{2} \leqq 1\right\} \text { and } \quad x_{1}-x_{2} \geqq s,
$$

with $x_{1}$ minimized. The random variables $s$ has a uniform distribution on $[0,1]$. The corresponding problem $\mathbf{D}$ obtained from this linear stochastic program is given by $(1.21), \cdots,(1.23)$ which becomes here: Find $\nu \in R, \pi(s)=\left(\pi_{1}(s), \pi_{2}(s)\right) \in \mathscr{L}_{2}^{1}$ satisfying

$$
-\nu+E\left\{\pi_{1}(s)\right\} \leqq 1, \quad \sigma \geqq 0
$$

almost surely

$$
\pi_{1}(s)+\pi_{2}(s) \geqq 1, \quad \pi_{1}(s) \geqq 0, \quad \pi_{2}(s) \geqq 0
$$

and maximizing

$$
-\nu+E\left\{\pi_{1}(s) \cdot s-\pi_{2}(s)\right\}
$$


It obvious that the optimal solution will have $\pi_{2}(s)=0$ a.s. and since $\pi_{1}(s) \geqq 0$ it follows that the constraint $\pi_{1}(s)+\pi_{2}(s) \geqq-1$ a.s. is never binding. Thus the optimal solution will have $\nu=\operatorname{Max}\left[0, E\left\{\pi_{1}(s)\right\}-1\right]$. Examining both cases it is clear that $\nu$ must be $=0$ in an optimal solution. Thus the problem $\mathbf{D}$ is equivalent to finding $\pi_{1}(s) \in \mathscr{L}^{1}$ such that

$$
\pi_{1}(s) \geqq 0 \quad \text { a.s. and } \quad E\left\{\pi_{1}(s)\right\} \leqq 1
$$

which maximize $E\left\{\pi_{1}(s) \cdot s\right\}$. It is easy to verify that the maximum is not attained by an $\mathscr{L}^{1}$ function. The sequence of functions

$$
\pi_{1 n}(s)=\left\{\begin{array}{lll}
0 & \text { for } & 0 \leqq s \leqq 1-\frac{1}{n} \\
n & \text { if } & 1-\frac{1}{n}<s \leqq 1
\end{array}\right.
$$

is a sequence of feasible solutions such that

$$
\lim _{n \rightarrow \infty} E\left\{\pi_{1 n}(s) \cdot s\right\}=\lim _{n \rightarrow \infty} 1-\frac{1}{2 n}=1=\sup \mathbf{D} .
$$

But the cluster point of the sequence $\left\{\pi_{1 n}\right\}$ is not in $\mathscr{L}^{1}$.

\section{REFERENCES}

1. A. Brøndsted and R. T. Rockafellar, On the subdifferentiability of convex functions, Proc. Amer. Math. Soc., 16 (1965), 605-611.

2. M. Eisner and P. Olsen, Duality for Stochastic Programming interpreted as L.P. in $L_{p}$ Space, Technical Report $n^{\circ}$ 201, Cornell University, Dept. Operations Research (1973).

3. S. Gartska, A Remark pertaining to Duality in Stochastic Programming with Recourse, Z. für Wahrscheinlichkeitstheorie ver. Gebiete (to appear).

4. P. Kall, Some remarks on the distribution problem of stochastic linear programming, Operations Research Verfahren, 16 (1972), 189-196.

5. A. Madansky, Dual variables in two-stage linear programming under uncertainty, J. Math. Anal. Appl., 6 (1963), 98-108.

6. P. Olsen, Measurability in Stochastic Programming, Technical Report n 196, Cornell University, Dept. Operations Research (1973).

7. R. T. Rockafellar, Convex Analysis, Princeton University Press, 1970.

8. — Conjugate Duality and Optimization, SIAM monograph series, 1974.

9. - Measurable dependence of convex sets and functions on parameters, J. Math. Anal. Appl., 28 (1969), 4-25.

10. - Integrals which are convex functionals, Pacific J. Math., 24 (1968), 525-540.

11. - Integrals which are convex functionals, II, Pacific J. Math., 39 (1971), 439-469. 
12. Characterization of the subdifferentials of convex functions, Pacific J. Math., 17 (1966), 497-510.

13. R. Wets, On inf-compact mathematical programs, Proceedings 5th IFIP Conference on Optimization, Springer-Verlag (1974). To appear.

14. - Problèmes duaux en programmation stochastique, C. R. Acad. Sc. Paris, 270 (1970), $47-50$.

Received March 5, 1975. The first author was supported in part by the Air Force Office of Scientific Research Air Force Systems Command, USAF, under grant AF-AFOSR-72-2269 and the second author was supported in part by the National Science Foundation under grant GP-31551.

UNIVERSITY OF WASHINGTON

AND

UNIVERSITY OF KENTUCKY 




\section{Pacific Journal of Mathematics}

\section{Vol. 62, No. $1 \quad$ January, 1976}

Mieczyslaw Altman, Contractor directions, directional contractors and

directional contractions for solving equations . .................. 1

Michael Peter Anderson, Subgroups of finite index in profinite groups .........

Zvi Arad, Abelian and nilpotent subgroups of maximal order of groups of odd order

John David Baildon and Ruth Silverman, On starshaped sets and Helly-type theorems ..........................................

John W. Baker and R. C. Lacher, Some mappings which do not admit an

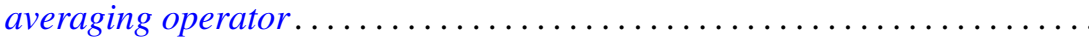

Joseph Barback, Composite numbers and prime regressive isols . . . . . . . . . .

David M. Boyd, Composition operators on $H^{p}(A) \ldots \ldots \ldots \ldots \ldots \ldots \ldots$

Maurice Chacron, Co-radical extension of PI rings . . . . . . . . . . . . .

Fred D. Crary, Some new engulfing theorems . . . . . . . . . . . . . . .

Victor Dannon and Dany Leviatan, A representation theorem for convolution transform with determining function in $L^{p} \ldots \ldots \ldots \ldots \ldots \ldots \ldots \ldots \ldots \ldots \ldots \ldots \ldots \ldots$

Mahlon M. Day, Lumpy subsets in left-amenable locally compact

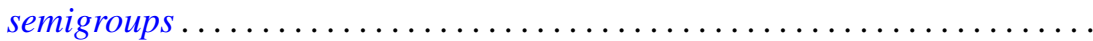

Michael A. Gauger, Some remarks on the center of the universal enveloping algebra of a classical simple Lie algebra . .

David K. Haley, Equational compactness and compact topologies in rings

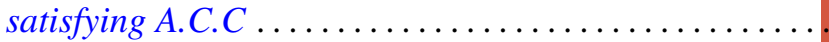

Raymond Heitmann, Generating ideals in Prüfer domains .

Gerald Norman Hile, Entire solutions of linear elliptic equations with

Laplacian principal part. .

Richard Oscar Hill, Moore-Postnikov towers for fibrations in which $\pi_{1}$ (fiber) is non-abelian

John Rast Hubbard, Approximation of compact homogeneous maps . .

Russell L. Merris, Relations among generalized matrix functions . .

V. S. Ramamurthi and Edgar Andrews Rutter, On cotorsion radicals ...

Ralph Tyrrell Rockafellar and Roger Jean-Baptiste Robert Wets, Stochastic

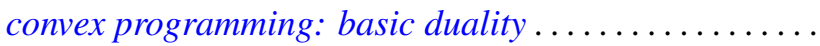

Alban J. Roques, Local evolution systems in general Banach spaces ..

I. Bert Russak, An indirect sufficiency proof for problems with bounded state variables.

Richard Alexander Sanerib, Jr., Ultrafilters and the basis property. .

H. A. Seid, The decomposition of multiplication operators on $L_{p}$-spaces . .

Franklin D. Tall, The density topology .................. 